\title{
Coherent soft x-ray pulses from an echo-enabled harmonic generation free-electron laser
}

Primož Rebernik Ribič, Alessandro Abrami, Laura Badano, Maurizio Bossi, Hans-Heinrich Braun, Niky Bruchon, Flavio Capotondi, Davide Castronovo, Marco Cautero, Paolo Cinquegrana, Marcello Coreno, Marie Emmanuelle Couprie, Ivan Cudin, Miltcho Boyanov Danailov, Giovanni De Ninno, Alexander Demidovich, Simone Di Mitri, Bruno Diviacco, William M. Fawley, Chao Feng, Mario Ferianis, Eugenio Ferrari, Laura Foglia, Fabio Frassetto, Giulio Gaio, David Garzella, Amin Ghaith, Fabio Giacuzzo, Luca Giannessi, Vanessa Grattoni, Sandi Grulja, Erik Hemsing, Fatma lazzourene, Gabor Kurdi, Marco Lonza, Nicola Mahne, Marco Malvestuto, Michele Manfredda, Claudio Masciovecchio, Paolo Miotti, Najmeh S. Mirian, Ivaylo Petrov Nikolov, Giuseppe Maria Penco, Gregory Penn, Luca Poletto, Mihai Pop, Eduard Prat, Emiliano Principi, Lorenzo Raimondi, Sven Reiche, Eléonore Roussel, Roberto Sauro, Claudio Scafuri, Paolo Sigalotti, Simone Spampinati, Carlo Spezzani, Luca Sturari, Michele Svandrlik, Takanori Tanikawa, Mauro Trovó, Marco Veronese, Davide Vivoda, Dao Xiang, Maurizio Zaccaria, Dino Zangrando, Marco Zangrando, and Enrico Massimiliano Allaria

\section{Abstract}

X-ray free electron lasers (FELs), which amplify light emitted by a relativistic electronbeam, are extending nonlinear optical techniques to shorter wavelengths, adding element specificity by exciting and probing electronic transitions from core levels. These techniques would benefit tremendously from having a stable FEL source, generating spectrally pure and wavelength-tunable pulses. We show that such requirements can be met by operating the FEL in the so-called echo-enabled harmonic generation (EEHG) configuration. Here, two external conventional lasers are used to precisely tailor the longitudinal phase space of the electron-beam prior to emission of $x$-rays. We demonstrate high-gain EEHG lasing producing stable, intense, nearly fully coherent pulses at wavelengths as short as $5.9 \mathrm{~nm}(\sim 211 \mathrm{eV})$ at the FERMI FEL user facility. Low sensitivity to electron-beam imperfections and observation of stable, narrow-band, coherent emission down to $2.6 \mathrm{~nm}(\sim 474 \mathrm{eV})$ make the technique a prime candidate for generating laser-like pulses in the $x$-ray spectral region, opening the door to multidimensional coherent spectroscopies at short wavelengths.

\section{Introduction}

Modern, high-gain FELs routinely produce femtosecond pulses with gigawatt peak power and tunable wavelengths ranging from several tens of $\mathrm{nm}$ to less than $0.1 \mathrm{~nm}$ (i.e., photon energies from several tens of $\mathrm{eV}$ up to $20 \mathrm{keV})^{1,2}$. Most employ the mechanism of selfamplified spontaneous emission (SASE) ${ }^{3-7}$, where the initial radiation seed arises from temporally and spectrally chaotic spontaneous emission in a periodic magnetic device called an undulator. This chaotic start leads to a highly fluctuating output, characterized by a relatively broad and noisy spectrum. Self-seeding techniques ${ }^{8,9}$ can be used to improve the 
spectral quality of a SASE FEL but only at the expense of intensity fluctuations, as the seed starts from noise.

Many limitations of a SASE FEL can be overcome by using a stable external seed to trigger the amplification process. Direct seeding schemes ${ }^{10-12}$, where the seed wavelength matches that of the FEL, are typically limited to wavelengths above several tens of $n m$, due to the lack of intense and coherent sources in the $x$-ray region. Coherent output at shorter wavelengths requires a frequency up-conversion technique such as high-gain harmonic generation $(\mathrm{HGHG})^{13,14}$. In this case, an optical laser is used to periodically modulate the energy of the electrons along the beam as they traverse a short undulator. Then the electron-beam passes through a magnetic chicane, where the purely sinusoidal energy modulation is transformed into a periodic density modulation that contains significant higher harmonic frequency components. The microbunched electron-beam finally emits coherent light at one or more preferred harmonics of the seed laser frequency, corresponding to the FEL resonances of the downstream undulators (also called radiator sections). The technique offers a natural way to produce highly coherent FEL pulses ${ }^{15}$ with tunable spectrotemporal properties ${ }^{16,17}$, and allows fine control over the output wavelengths in two-colour operation ${ }^{18,19}$.

In HGHG, the required energy modulation amplitude for producing substantial bunching scales linearly with the harmonic number $h$. For very high harmonics, the strong energy modulation severely diminishes the FEL gain in the downstream radiator, typically limiting the scheme to $h \lesssim 15$, corresponding to FEL wavelengths of about $10-20 \mathrm{~nm}^{15}$ if a UV seed generated by a solid state optical laser is used. A two-stage (cascade) HGHG setup based on the fresh-bunch (FB) approach ${ }^{20}$ extends the harmonic range up to $h \sim 60-70^{21}$. However, at such high $h$, the sensitivity to the initial electron-beam phase space becomes critical and may severely affect the FEL radiation in terms of temporal coherence, pulse energy, and shot-to-shot stability. Furthermore, the scheme cannot cover the whole harmonic range, as the final harmonic number is a product between the harmonic numbers of the individual stages. In addition, the FB setup uses a relatively large portion of the electron-beam to accommodate the two stages and makes the implementation of double-pulse operation more difficult.

The drawbacks of two-stage HGHG can be overcome by using a recently proposed technique called echo-enabled harmonic generation $(\mathrm{EEHG})^{22,23}$, where the electron-beam longitudinal phase space is precisely shaped using two seed lasers to generate bunching at high harmonics. The method requires a much weaker energy modulation compared to HGHG and is also intrinsically much less sensitive to the initial electron-beam imperfections ${ }^{24}$, making it a strong candidate for producing highly stable, nearly fully coherent, and intense FEL pulses down to soft $x$-ray wavelengths.

\section{The EEHG scheme}

The EEHG layout is shown in Fig. 1. Two modulating sections, each consisting of a seed laser, short magnetic undulator (modulator) and a magnetic chicane, are used to manipulate the electron-beam phase space prior to injecting the electrons into the radiator. As a result, the electron-beam coming out of the second chicane is density-modulated at wavenumbers 
given by $k_{E}=n k_{1}+m k_{2}$, where $n$ and $m$ are non-zero integers and $k_{1,2}=2 \pi / \lambda_{1,2}$ are the wavenumbers of the two seed lasers operating at wavelengths $\lambda_{1}$ and $\lambda_{2}$. In the case shown below (and in the experiment reported here), $\lambda_{1}=\lambda_{2}$, so $k_{E}=(n+m) k_{1}$, or $\lambda_{E}=\lambda_{1} / h$, where $h=n+m$ is the harmonic number and $\lambda_{E}$ is the output modulation wavelength. By properly tuning the energy modulations and chicane strengths, only one set of $(n, m)$ pairs, where $n$ is typically small and negative and $m$ is large and positive, significantly contributes to the density modulation at a given harmonic $h^{23,24}$. To optimize bunching at a specific $h$, the dispersive strength of the $2^{\text {nd }}$ chicane is set approximately to $R_{56}^{(2)}=\frac{|n|}{h} R_{56}^{(1)}$, where $R_{56}^{(1)}$ is the $1^{\text {st }}$ dispersive strength. In this optimized condition, the bunching is relatively insensitive to the energy modulation $\Delta E_{1}$ produced by the $1^{\text {st }}$ seed laser. On the other hand, the required energy modulation amplitude from the $2^{\text {nd }}$ seed scales as $\Delta E_{2} \sim 1 / R_{56}^{(2)}$. Analysis also shows ${ }^{23}$ that bunching is maximized for $n=-1$. However, if $R_{56}^{(1)}$ is limited due to hardware constraints (as in our case), this scenario requires a low $R_{56}^{(2)}$ for high harmonics, which in turn calls for high $\Delta E_{2}$. As in standard HGHG operation, the increased energy spread will decrease the FEL gain. For the studies reported here, it was therefore beneficial to work at $n \leq-2$ for $h \gtrsim 45$.

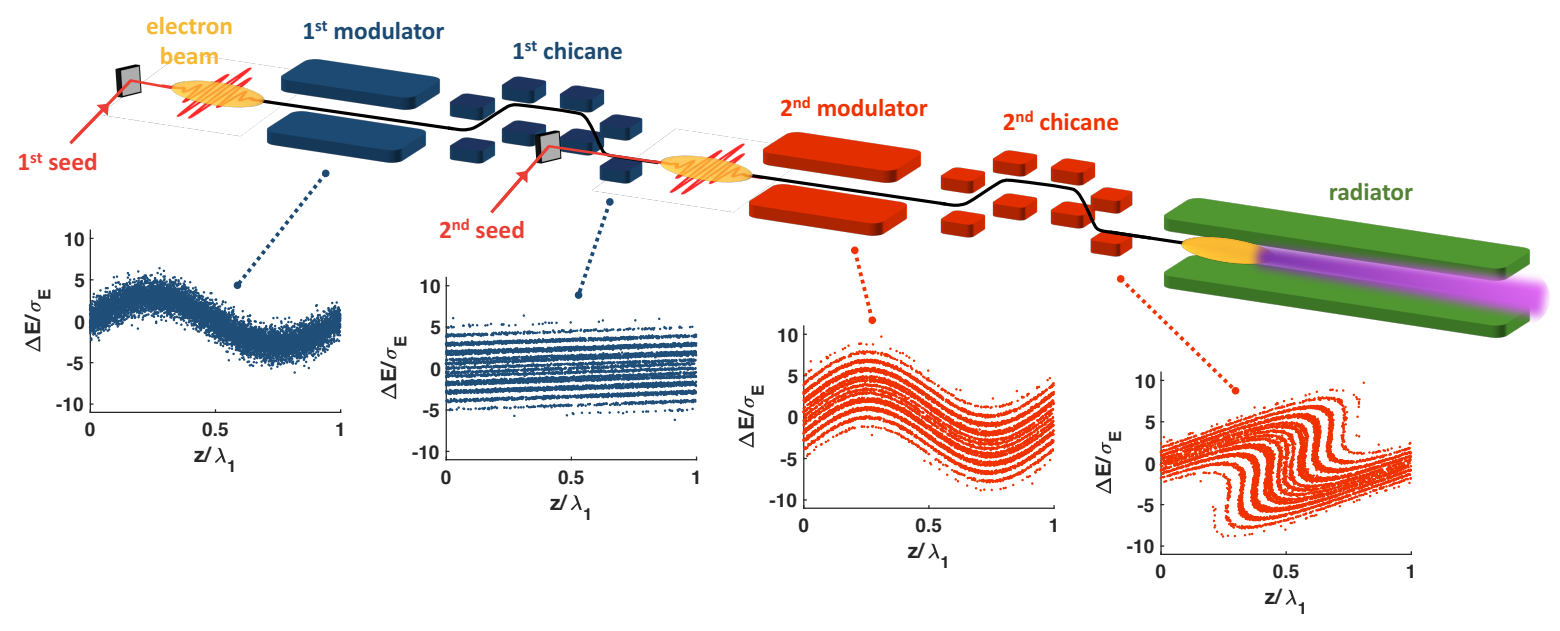

Figure 1: The EEHG scheme together with the electron-beam longitudinal phase space at different stages of the evolution. The $1^{\text {st }}$ seed laser with a wavelength $\lambda_{1}$ imprints a sinusoidal energy modulation with an amplitude $\Delta E_{1} \lesssim 3 \sigma_{E}$, where $\sigma_{E}$ is the initial uncorrelated energy spread, onto the relativistic electron-beam in the $1^{\text {st }}$ modulator. After passing through a strong $1^{\text {st }}$ chicane, the electrons with different energies move relative to each other according to $\Delta \mathrm{z}=R_{56}^{(1)} \Delta E / E_{0}$, where $\Delta \mathrm{z}$ is the displacement of a particle with an energy offset of $\Delta E$ with respect to a reference particle with an energy $E_{0}$. Such particle motion results in a striated phase space with multiple energy bands. The electrons then pass through the $2^{\text {nd }}$ modulator, where their energy is again periodically modulated using a $2^{\text {nd }}$ seed laser with $\lambda_{2}=\lambda_{1}$ (used in our experiment but not a requirement of EEHG) and $\Delta E_{2} \sim \Delta E_{1}$. After traversing a weaker $2^{\text {nd }}$ chicane, the electron-beam phase space is rotated, transforming the sinusoidal energy modulation into a periodic density modulation with high frequency components. As the energy spread within a single band is much smaller than $\sigma_{E}$, only a moderate $\Delta E_{2}$ is required to reach very high harmonics. The electron-beam is then 
injected into the radiator (a long undulator), tuned to emit light at a high harmonic of the $2^{\text {nd }}$ seed laser.

Early proof-of-principle EEHG experiments demonstrated coherent emission ${ }^{25}$ and later FEL amplification ${ }^{26}$ at low $\left(3^{\text {rd }}\right.$ and $4^{\text {th }}$ ) harmonics of an infrared (IR) seed. In follow-on studies $^{27,28}$, the harmonic range of coherent emission was progressively extended to $h=7$ and $h=15$, again using an IR seed laser. A recent demonstration ${ }^{29}$ of coherent emission at $h \sim 75$ of a seed with a wavelength of $2400 \mathrm{~nm}$ has shown that the technique could in principle be used to generate intense, fully coherent and tunable soft x-rays with UV lasers. However, up to now neither FEL amplification nor coherent emission was reported in the soft-x-ray spectral region. In this regime, seed laser phase errors ${ }^{30}$ as well as electron-beam instabilities ${ }^{31}$ can strongly affect the emission process and may limit the effectiveness of the EEHG scheme at very high harmonics.

Here, we demonstrate the first high-gain lasing of an EEHG FEL in the soft-x-ray region at 7.3 $\mathrm{nm}(\sim 169 \mathrm{eV})$ and $5.9 \mathrm{~nm}(\sim 211 \mathrm{eV})$, i.e., the $36^{\text {th }}$ and $45^{\text {th }}$ harmonics of the seed wavelength $\lambda_{1}=264 \mathrm{~nm}$ (Methods 2). We measure coherent emission at wavelengths as short as $2.6 \mathrm{~nm}(\sim 474 \mathrm{eV})$, demonstrating that significant bunching could be produced up to harmonics as high as 101 . This suggests it will be possible to extend the lasing region in EEHG-seeded FELs down to the water window ( $\sim 4 \mathrm{~nm}$ or $\sim 310 \mathrm{eV})$ and beyond, to the oxygen $\mathrm{K}$ edge $(\sim 2.3 \mathrm{~nm}$ or $\sim 540 \mathrm{eV})$. The experiment was performed at the FERMI FEL user facility in Trieste, Italy.

\section{Results}

The left panel of Fig. 2 shows the gain curve (power build-up) of the FERMI FEL ${ }^{15,21}$ in EEHG mode at $\lambda=7.3 \mathrm{~nm}(\sim 169 \mathrm{eV})$, i.e., $h=36$, measured by setting one-by-one each of the six radiator sections to the resonant condition. The electron-beam (Methods 1 ) was accelerated to a nominal energy of $E_{0}=1.31 \mathrm{GeV}$ in a linear accelerator (linac) and sent to the EEHG beamline (Fig.1 and Methods 3). The longitudinal dispersion of the strong (first) chicane was fixed at $2.38 \mathrm{~mm}$, while that of the weaker (second) chicane was set to $62 \mu \mathrm{m}$ to optimize bunching at $h=36$ in the $n=-1$ tune. To maximise gain, the six FERMI FEL radiator sections ${ }^{21}$ were operated in circular polarisation.
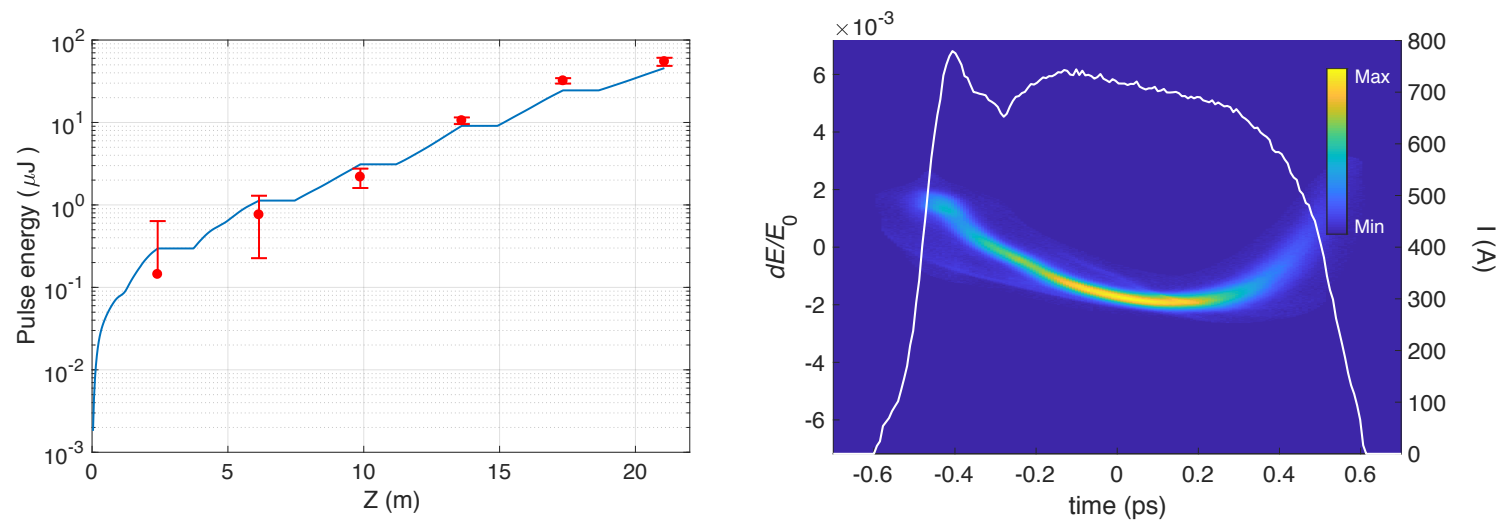
Figure 2: EEHG gain curve at $7.3 \mathrm{~nm}(\sim 169 \mathrm{eV})$ and typical electron-beam longitudinal phase space at the FERMI FEL. Left: Simulated (blue line) and rescaled (red circles) FEL pulse energy, obtained from measurements along the radiator. The pulse energy after each radiator section was determined from spectrometer images after background subtraction and calibrated with a photodiode. The error bars represent RMS intensity fluctuations. These values were then multiplied by a factor of 2 to match the simulations (see text for details). The input parameters for the Genesis ${ }^{32}$ simulation were extracted from the measured phase space, Right, giving typical values of $700 \mathrm{~A}$ for the current and $150 \mathrm{keV}$ for the uncorrelated energy spread $\sigma_{E}$ in the central region of the beam. The electron-beam energy profile shows a residual quadratic energy chirp $\left(\sim 20 \mathrm{MeV} / \mathrm{ps}^{2}\right)$ induced by longitudinal wake-fields during acceleration in the FERMI linac ${ }^{33}$. The right axis refers to the current profile (white line) and the left to the energy profile. The charge density is shown in false colours.

The gain curve displays exponential growth with a gain length $L_{G}=1.9 \mathrm{~m}$ and some indications of saturation in the last radiator segment. Genesis ${ }^{32}$ simulations (Methods 5) using experimentally determined parameters of the two seed lasers and electron-beam parameters extracted from the measured longitudinal phase space in the right panel of Fig.2 show a matching gain length. To compare the experimental and simulated gain lengths, the measured pulse energies at the detector were multiplied by a factor of 2 to match the simulation. Part of the discrepancy between the measured values and the ones predicted from simulations may be associated to a degradation of the transmission efficiency of the beamline optics and detectors, but we believe that the simulation may also overestimate the effective bunching at the radiator entrance, since it does not account for any bunching degradation in the strong chicane nor that due to phase distortions of the second seed laser transverse wavefront, which may become critical at high harmonics. We estimated the peak output power at the photodiode detector to $420 \mathrm{MW}$ by dividing the measured pulse energy of $25 \mu \mathrm{J}$ by the estimated pulse duration of $60 \mathrm{fs}$, obtained from simulation or by performing the Fourier transform of the bunching spectral distribution (Methods 6 ).

The sensitivity of the EEHG output to electron-beam properties was evaluated by recording the FEL spectra as a function of the delay between the electron-beam and the two seed pulses (varying their arrival time with respect to the electron-beam, maintaining their temporal overlap) and was compared to that of the two-stage HGHG based on the FB approach. The results are shown in Fig.3. To enable a quantitative comparison, we performed the experiment at the same FEL wavelength of $8.8 \mathrm{~nm}(\sim 141 \mathrm{eV})$, i.e., $h=30$, using the same electron-beam (which required reducing its energy to $1.1 \mathrm{GeV}$, with other parameters remaining similar to the ones in Fig.2). While EEHG shows an almost flat response, the central wavelength for the HGHG mode shifts a few times its spectral width during a $500 \mathrm{fs}$ scan of the seed laser arrival time. In addition, the central wavelength at a fixed delay (bottom panels) is nearly constant for EEHG, while it fluctuates by approximately one spectral width for HGHG. HGHG spectra also display a wider and more irregular shape. 

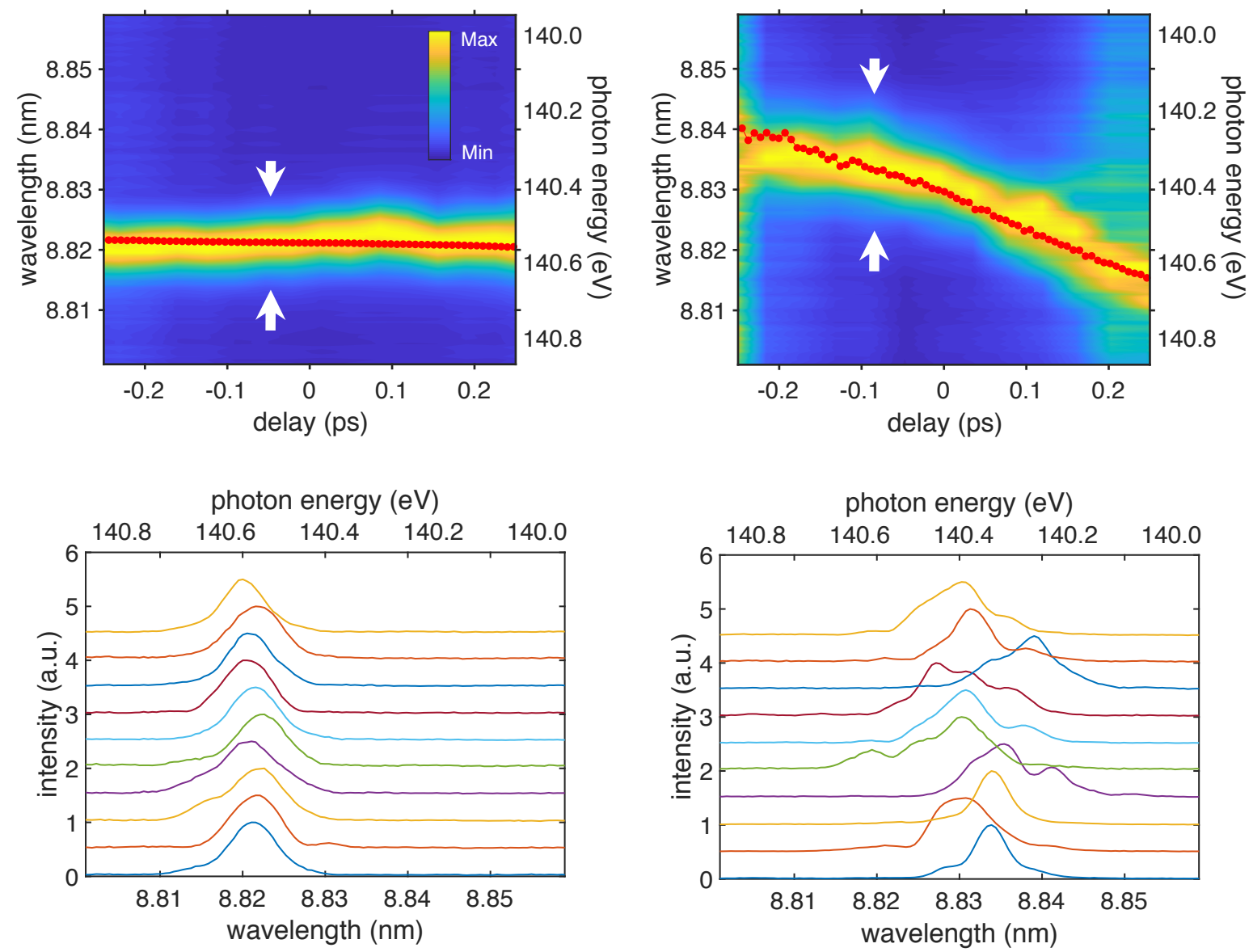

Figure 3: Sensitivity of the FEL output to the electron-beam properties. Left column: EEHG, right column: two-stage HGHG. Top row: Averaged and normalized (for clarity) FEL spectrum as a function of the delay between the electron-beam and the seed(s). For two-stage HGHG, the whole $500 \mathrm{fs}$ region where FEL emission was observed is shown. For EEHG, the duration of the scan was adjusted to the same as that of HGHG (for a clear comparison) and was chosen to contain the region of maximum FEL intensity. The red dots show the calculated central FEL wavelength using the measured electron-beam local linear energy chirp (see text). Bottom row: Consecutive (normalized) single-shot spectra taken at the delay near the maximum intensity, which is indicated by white arrows in the top panels.

The plots in Fig. 3 can be explained by considering the phase variation $\Delta \psi \approx$ $-k_{E}\left(R_{56}^{(2)}+\frac{n}{h} R_{56}^{(1)}\right) \frac{\Delta E}{E_{0}}$ of the EEHG bunching factor (Methods 6 ) accumulated in the magnetic chicanes due to the varying electron-beam energy profile ${ }^{24,34}$. Here, $\Delta E$ is the deviation from the nominal electron-beam energy $E_{0}$. For a beam with a varying local linear energy chirp $\frac{d E}{d t}(t)$, i.e., varying slope of the longitudinal phase space in the right panel of Fig. 2, this gives a relative shift of the central bunching wavelength $\frac{d \lambda}{\lambda}(\tau) \approx-\frac{1}{E}\left(R_{56}^{(2)}+\right.$ $\left.\frac{n}{h} R_{56}^{(1)}\right) \frac{d E}{c d t}(\tau)$, which depends on the position $\tau$ of the seed (i.e., the delay in Fig.3) with respect to the electron-beam. When EEHG is optimized, it operates in a condition where the ratio between the $2^{\text {nd }}$ and $1^{\text {st }}$ dispersive strengths is approximately $\frac{|n|}{h}$, with $n<0$, leading 
to a near cancellation of the terms in the brackets in the expression for $d \lambda$, as well as in the more general expression for $\Delta \psi$. Therefore, although the local linear energy chirp is changing along the electron-beam, the scan in the top left panel of Fig. 3 shows an almost flat response. For HGHG the same formula can be used by setting the $1^{\text {st }}$ dispersion to zero. Hence, there is always a shift of the central wavelength in the presence of a linear energy chirp. For two-stage HGHG, the formula has to be applied twice, taking into account different dispersions for the two stages and the delay (FB approach) between the first and second stage emission ${ }^{34}$. The red dots in the top panels in Fig. 3 are the calculated central FEL wavelengths with the above formula using the measured linear chirp as the input parameter and show an excellent agreement with the measured spectra (fine structures in the delay scans such as, e.g., the bump around 0.1 ps in the top left panel of Fig. 3, cannot be reproduced using calculations due to the limited resolution of the electron-beam phase space measurement and may also be a consequence of small FEL pointing drifts). The same effect, combined with the electron-beam arrival time jitter ( $\sim 50 \mathrm{fs})$, also explains the fluctuating central wavelength for the HGHG operational mode. Higher order energy variations, which cannot be resolved in the measured phase space in Fig. 2 , further affect the phase of the bunching factor. These can be attributed to electron-beam instabilities ${ }^{35,36}$ and may vary shot-to-shot. While such variations minimally affect the EEHG bunching phase, they result in a more structured and fluctuating spectrum in the case of HGHG.

A detailed analysis of the FEL spectra (Methods 5$)$ in the EEHG mode at $\lambda=7.3 \mathrm{~nm}(\sim 169$ $\mathrm{eV})$, i.e., $h=36$ and $\lambda=5.9 \mathrm{~nm}(\sim 211 \mathrm{eV})$, i.e., $h=45$ in Fig. 4 shows a Gaussian-like shape in both cases with a relative central wavelength (photon energy) stability of $\sim 7 \times 10^{-5}$, and $16 \%$ and $25 \%$ RMS intensity fluctuations at $7.3 \mathrm{~nm}$ and $5.9 \mathrm{~nm}$, respectively. The minimum width containing $76 \%$ of the pulse energy $\left(\sigma_{76 \%}\right)$, was used as a measure of the spectral width. Such a definition, that matches the standard full-width half-maximum (FWHM) for a perfectly Gaussian curve, is more sensitive to the tails of the spectrum (compared to the FWHM), making it possible to account for spectral features coming from electron-beam imperfections and laser phase errors. The measured average $\sigma_{76 \%}$ at $7.3 \mathrm{~nm}$ $(\sim 169 \mathrm{eV})$ is $3.0 \times 10^{-3} \mathrm{~nm}(70 \mathrm{meV})$, giving a relative bandwidth of $\sim 4 \times 10^{-4}$. This is approximately $1.5 \times$ the $\sigma_{76 \%}$ calculated from the bunching spectral envelope, assuming flat phases for both seed lasers and a flat electron-beam energy profile (Methods 6 ). The deviations from the theoretical value are probably due to a residual linear frequency chirp on the $2^{\text {nd }}$ seed laser (the effect of the electron-beam quadratic energy chirp is negligible in our case), non-zero spectrometer resolution ( $\sim 20 \mathrm{meV})$ and possible spectral broadening due to electron-beam instabilities. At $\lambda=5.9 \mathrm{~nm}(\sim 211 \mathrm{eV})$, the measured average $\sigma_{76 \%}$ is $2.4 \times 10^{-3} \mathrm{~nm}(88 \mathrm{meV}), 1.8 \times$ the value obtained from the bunching envelope. The increased deviation from the calculated bandwidth (compared to $\lambda=7.3 \mathrm{~nm}$ ) is due to significant sideband structures in the spectra, as shown in the bottom left panel of Fig.4. We attribute this to a higher sensitivity of the bunching phase $\Delta \psi$ to electron-beam imperfections at shorter wavelengths. Indeed, the use of a laser heater ${ }^{37}$ was critical to suppress such electron-beam instabilities and obtain cleaner spectra. Furthermore, additional measurements show a correlation between the position and intensity of the spectral sidebands and electron-beam compression, confirming that spectral degradation is related to the electron-beam and not laser longitudinal phase errors ${ }^{30}$, even at these high harmonic numbers. 

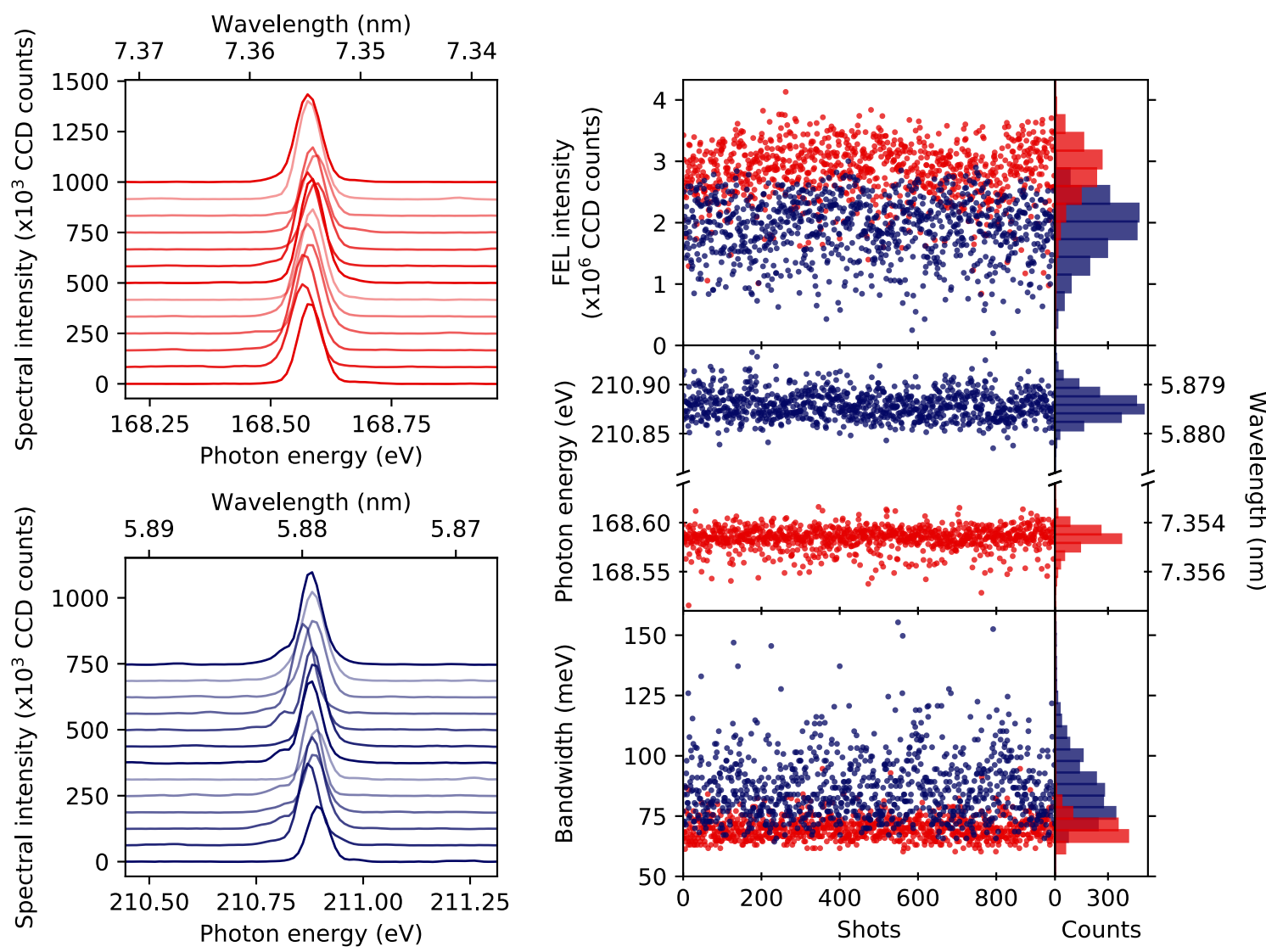

Figure 4: EEHG performance at the FERMI FEL in the soft-x-ray region. Left column: single shot spectra randomly chosen in a sequence of 2000 consecutive shots at (top) $\lambda=7.3 \mathrm{~nm}$ $(\sim 169 \mathrm{eV})$ and (bottom) $\lambda=5.9 \mathrm{~nm}(\sim 211 \mathrm{eV})$ in the $n=-1$ tune. Right column: data for $7.3 \mathrm{~nm}(\sim 169 \mathrm{eV})$ and $5.9 \mathrm{~nm}(\sim 211 \mathrm{eV})$ are shown in red and blue, respectively. FEL intensity (top), central wavelength/average photon energy (middle), and $\sigma_{76 \%}$ spectral width (bottom) for the 2000 consecutive shots and the corresponding histograms.

Benchmarking the above results against the nominal performances achieved with the twostage HGHG at the FERMI FEL showed that EEHG gives significantly better spectra in terms of central wavelength stability and bandwidth. This is especially true for high harmonics $(h=45)$, where the performance is affected by electron-beam imperfections, which have a stronger influence in the case of HGHG.

At $h>45$, the longer gain lengths did not allow reaching saturation by the radiator's end. Nevertheless, by increasing the electron-beam energy to $1.5 \mathrm{GeV}$, we could observe coherent harmonic emission with relatively clean spectra up to $h=101$, as shown in Fig. 5 . The left panel shows the spectrum at $\lambda=3.1 \mathrm{~nm}(\sim 394 \mathrm{eV})$, i.e., $h=84$. Due to the limited seed power and dispersive strength of the first chicane we had to operate EEHG at $n=-4$, resulting in reduced bunching. Within the resolution, the spectrum is a narrow single-line with relatively weak pedestal structures, even at this high harmonic number. The right panel shows EEHG in the $n=-4$ tune, optimized for emission at $\lambda=2.6 \mathrm{~nm}(\sim 474 \mathrm{eV})$, i.e., $h=$ 101. Due to the absence of gain, the EEHG signal becomes comparable to the broadband 
spontaneous emission coming from the whole electron-beam (see inset). This, in combination with a limited $1^{\text {st }}$ chicane strength and $2^{\text {nd }}$ seed laser power forcing operation at $n \leq-4$ with decreased bunching, prevented us from observing coherent emission at even higher harmonics.
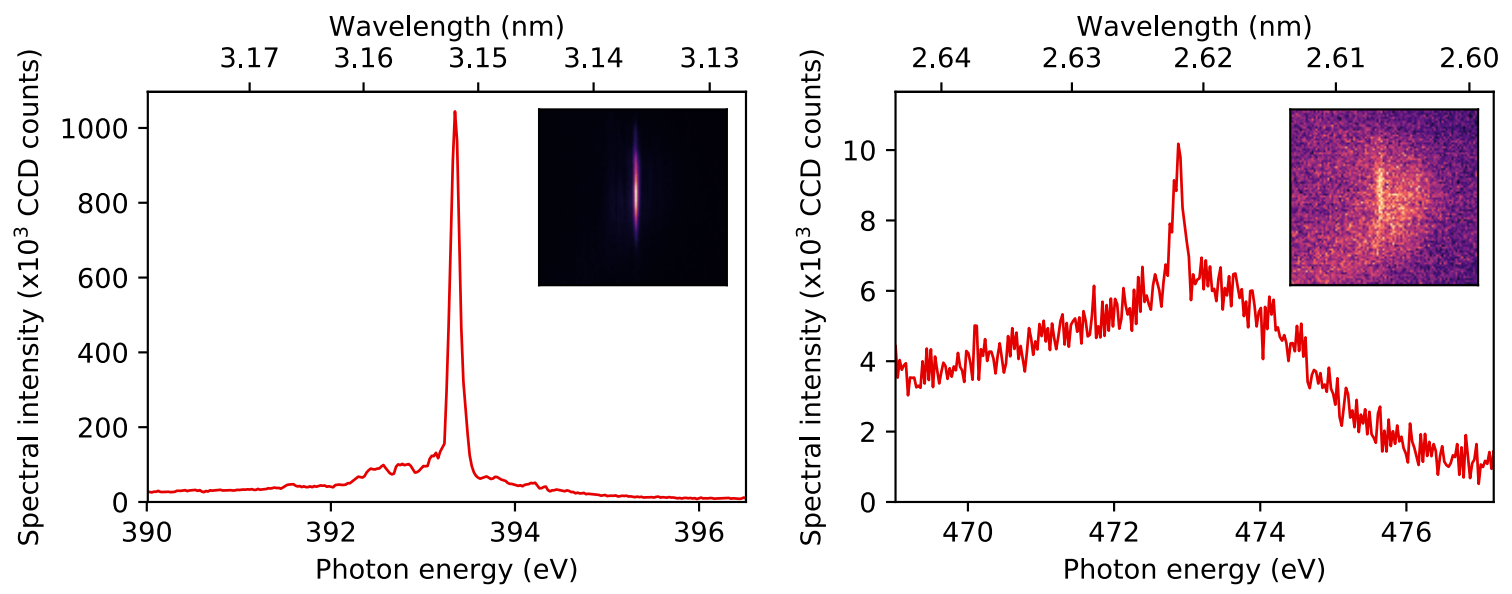

Figure 5: EEHG at high harmonics. Coherent emission spectra at $\lambda=3.1 \mathrm{~nm}(\sim 394 \mathrm{eV})$ (left) and $\lambda=2.6 \mathrm{~nm}(\sim 474 \mathrm{eV})$ (right). Insets show the raw CCD images. Spontaneous broadband emission (displaying its characteristic shape) is identified as the main contribution to the strong background at $2.6 \mathrm{~nm}$.

In EEHG, the bunching factor is significant also for harmonics lying close to the one that is optimized, thus in principle allowing multi-colour FEL operation for appropriate radiator configurations. Two-colour emission is demonstrated in Fig. 6, where we tuned the first four radiator sections to $\lambda=5.9 \mathrm{~nm}(\sim 211 \mathrm{eV})$, i.e., $h=45$ and the last two to $\lambda=5.7 \mathrm{~nm}$ $(\sim 216 \mathrm{eV})$, i.e., $h=46$, with EEHG operating in the $n=-2$ condition. The spectra show a Gaussian-like shape with similar statistics as in Fig. 4 but with decreased pulse energies, as only part of the radiator was used for amplification of each harmonic. It should be noted that multi-colour emission at short wavelengths is possible also in the two-stage HGHG operational mode at the FERMI FEL. However, the fact that cascaded HGHG cannot cover the whole harmonic range limits the tunability of this approach. 


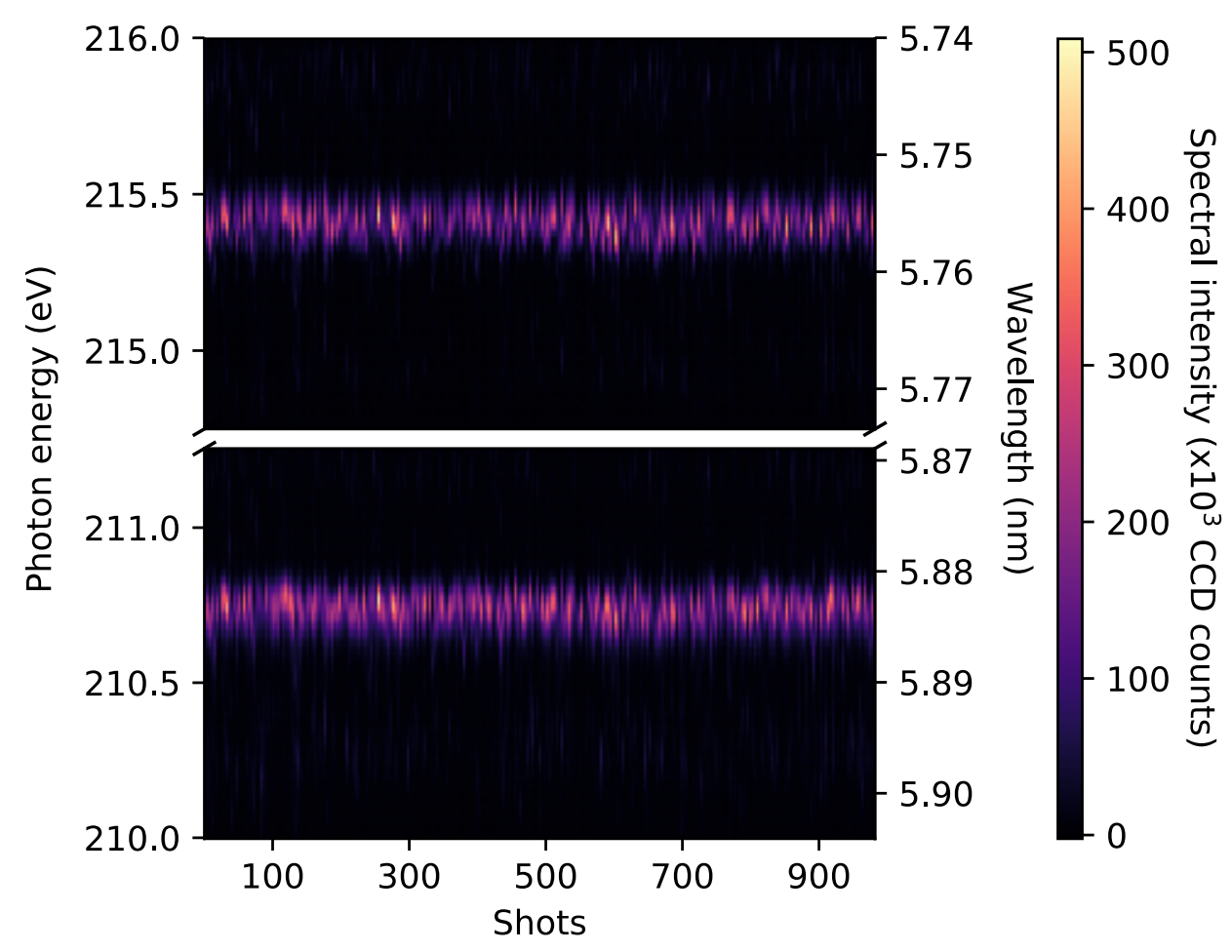

Figure 6: EEHG in two-colour operation. The scheme was optimized for emission around the $45^{\text {th }}$ harmonic with the first four radiator sections tuned to $\lambda=5.9 \mathrm{~nm}(\sim 211 \mathrm{eV})$ and the last two to $\lambda=5.7 \mathrm{~nm}(\sim 216 \mathrm{eV})$. The panels show 1000 consecutive single-shot spectra.

\section{Discussion}

We have demonstrated high-gain and high-quality lasing using EEHG at the FERMI FEL down to wavelengths as short as $5.9 \mathrm{~nm}(\sim 211 \mathrm{eV})$. In comparison to the output produced via a two-stage HGHG scheme, EEHG shows both narrower and cleaner spectra with significantly less shot-to-shot central wavelength jitter and the same time a comparable energy per pulse. The current limitations of the EEHG setup at FERMI (limited $1^{\text {st }}$ chicane strength and $2^{\text {nd }}$ seed laser power) prevented us from achieving higher initial bunching and from reaching saturation at shorter wavelengths. In this regime, the two-stage HGHG still outperforms EEHG in terms of energy per pulse. On the other hand, the observation of coherent emission at harmonics in the range from 84 to 101 indicates the possibility to extend the lasing to wavelengths as short as $2 \mathrm{~nm}(620 \mathrm{eV})$ or less in a more optimised setup, either by using EEHG directly, or with a cascade employing both EEHG and HGHG schemes ${ }^{24}$. Such a layout has the potential to set the stage for entirely new experiments using $\mathrm{x}$-ray nonlinear optical techniques $^{38-42}$.

One of the main advantages of EEHG over two-stage HGHG is multi-wavelength emission without the limitations due to the harmonic conversion of the first stage. This allows either the generation of two (or more) FEL pulses tuned across a given core-hole resonance and separated by the characteristic energy of a valence band excitation, or hitting core transitions of two distinct elements in the sample. Both configurations might be exploited in 


\author{
wave-mixing approaches, so far only theoretically evaluated ${ }^{43}$, for gaining information on \\ dynamics and correlations between valence excitations and selected atoms in an \\ experimental sample.
}

\title{
Methods
}

1. Electron beam. The electron-beam was generated by a photo-injector consisting of a radio-frequency (RF) photocathode gun and a booster linac ${ }^{44}$, compressed to $\sim 700 \mathrm{~A}$ by a magnetic chicane, and accelerated to 1.1 , 1.3 or $1.5 \mathrm{GeV}$ (depending on the FEL wavelength) by a RF linac ${ }^{45}$. A fourth-harmonic RF cavity was used to linearize the compression process and generate a nearly flat current profile. The slice normalised transverse emittances and energy spread at the linac end were $\approx 1 \mathrm{~mm}$ mrad and $\approx 150 \mathrm{keV}(\mathrm{rms})$, respectively ${ }^{46}$. During acceleration, the electron beam experienced nonlinear wakefields, resulting in a residual quadratic energy curvature of $\sim 20 \mathrm{MeV} / \mathrm{ps}^{2}$. The longitudinal phase space distribution at the end of the linac was measured by vertically stretching the electron-beam using an RF deflecting cavity and dispersing it horizontally with a dipole magnet ${ }^{47}$.

2. Seed lasers. The seed laser pulses were generated by a Ti:Sapphire laser system incorporating a single mode-locked oscillator and two separate regenerative amplifiers delivering infrared pulses in the $792 \mathrm{~nm}$ range with an energy of up to $5 \mathrm{~mJ}$ each at a repetition rate of $50 \mathrm{~Hz}$. The first amplifier output was frequencytripled and then sent to the insertion breadboard in front of the first modulator, while the IR pulse generated by the second amplifier was transported to an optical breadboard close to the second insertion point in the undulator hall, where the second UV pulse was generated. The duration, bandwidth, and chirp of both UV pulses were controlled by using different third-harmonic generation crystal sets and by UV grating compressors. The pulses used for the measurements reported here had a nearly Gaussian shape with a FWHM of 175 and $130 \mathrm{fs}$, respectively, and a nearly constant phase. The spot sizes in the first and second modulator were around 700 and 350 micrometers $1 / e^{2}$ diameter, respectively. The peak laser powers were estimated to be $43 \mathrm{MW}$ for the first and $90 \mathrm{MW}$ for the second seed pulse, resulting in energy modulation amplitudes equal to a few times $(\lesssim 3)$ the initial uncorrelated electron-beam energy spread.

3. EEHG beamline. The FEL-2 beamline of FERMI ${ }^{21}$ had to be modified to allow operation in the EEHG mode. The existing delay line was used as the $1^{\text {st }}$ (strong) chicane. For this purpose, the positions of its dipole magnets were modified and a new power supply was installed to increase the dispersion up to $2.4 \mathrm{~mm}$ for energies around $1.3 \mathrm{GeV}$. The $2^{\text {nd }}$ stage modulator had to be replaced with a longer-period undulator (13 periods with a period length of $11.3 \mathrm{~cm}$ ) so that its resonance could be tuned to $264 \mathrm{~nm}$. A new laser injection system together with various laser and electron-beam diagnostics was installed in the delay line chicane for injecting the $2^{\text {nd }}$ seed laser beam and for performing the transverse overlap between the beams. Other components, i.e., $1^{\text {st }}$ seed laser, $1^{\text {st }}$ modulator ( 30 periods with a period length of $10 \mathrm{~cm}$ ), $2^{\text {nd }}$ chicane, and the radiator ( 6 sections with 68 periods each and a period length of $3.5 \mathrm{~cm}$ ) were not modified. The first stage radiator gaps were completely opened so as not to interfere with the bunching process. The average electronbeam beta function along the beamline was $\approx 10 \mathrm{~m}$.

4. Diagnostics. FEL spectra were measured using a dedicated spectrometer ${ }^{48}$. The photon beam was diffracted by a planar variable-spacing grating, which focused the first-order diffraction mode onto a YAG screen. The resulting fluorescence was imaged by a charge-coupled device (CCD). A photodiode was used to calibrate the intensity. The setup allowed detection of single-shot spectra for FEL pulse energies higher than a few $\mu \mathrm{J}$. The relatively low intensity produced at harmonics 84 and 101 could not be detected with the standard spectrometer. For these two wavelengths, an in-vacuum CCD was used to directly detect the soft-x-ray photons diffracted by the spectrometer grating. Measurements at $2.6 \mathrm{~nm}$ required operating the spectrometer at the second diffraction order. In all cases, the spectra (e.g., CCD counts vs. wavelength in Fig. 4, left panels) were obtained by projecting the CCD images onto the horizontal spectrometer axis. The FEL intensity (CCD counts) in the top right panel of Fig. 5 was obtained by an additional integration over the wavelength axis of the spectrometer. In Fig. 5 we included the raw CCD data as an inset to show the characteristic shape of broadband spontaneous emission from the undulators. 
5. Simulations. We simulated the performance of the EEHG scheme from the first modulator entrance to the radiator exit with the code Genesis $1.3^{32}$. To increase the accuracy of the results, one-to-one calculations in which every beam electron is considered were performed. The simulations did not include collective effects such as coherent and incoherent synchrotron radiation nor intra-beam scattering. Experimentally determined parameters listed in the previous sections were used as the input parameters for the simulation. As in the experiment, the power of the second seed laser, the strength of the second dispersive section and the magnetic field of the radiator were optimized to obtain FEL radiation with high pulse energies and clean spectra.

6. Spectral distribution from bunching. The EEHG spectral distribution was calculated from the bunching factor, which for a general electron-beam energy profile $E(t)=E_{0}+\Delta E(t)$, where $E_{0}$ is the nominal electron-beam energy, and arbitrary (slowly varying) seed laser energy modulation amplitudes $A_{1,2}(t)=$ $\Delta E_{1,2}(t) / \sigma_{E}$ and phases $\psi_{1,2}(t)$ along the electron-beam longitudinal coordinate $t$ reads ${ }^{23}$ :

$b(k)=e^{-\frac{1}{2}\left[\xi+\frac{\Delta k}{k_{1}} B\right]^{2}} \int_{-\infty}^{\infty} J_{m}\left[-\frac{k}{k_{1}} B_{2} A_{2}(t)\right] \times J_{n}\left[-\left(\xi+\frac{\Delta k}{k_{1}} B\right) A_{1}(t)\right] e^{-i\left(\frac{k}{k_{1}} B-K m B_{1}\right) \frac{\Delta E(t)}{\sigma_{E}}} e^{i\left(n \psi_{1}(t)+m \psi_{2}(t)\right)} e^{-i \Delta k c t} c d t$

where $\xi=n B_{1}+(n+K m) B_{2}$ is the EEHG scaling parameter, $\Delta k=k-k_{E}, B_{1,2}=R_{56}^{1,2} k_{1} \sigma_{E} / E_{0}$ are the dimensionless dispersive strengths and $B=B_{1}+B_{2}, k_{1,2}=2 \pi / \lambda_{1,2}$ are the wavenumbers of the two seeds and $K=k_{2} / k_{1}$. We compared the measured $\sigma_{76 \%}$ bandwidth with the one calculated for a flat electron-beam energy profile, constant seed laser phases, and Gaussian seed envelopes with parameters given in the Seed lasers section. The small quadratic electron-beam chirp (Fig. 2, right panel) cannot explain the deviations from the measured spectral width in our case. However, including a residual chirp on the $2^{\text {nd }}$ seed laser significantly affects the spectral distribution; e.g., a group delay dispersion of $2 \times 10^{-27} \mathrm{~s}^{2}$ would stretch the $2^{\text {nd }}$ seed duration by only $5 \%$, but would increase the FEL bandwidth by $30 \%$. Such a linear frequency chirp on the seed laser can in principle be fully compensated by a suitable compressor. Additional spectral broadening comes from the spectrometer resolution and possible electron-beam instabilities that contribute to the bunching phase. The modulation amplitude of the $2^{\text {nd }}$ seed was determined by maximizing the time independent bunching factor ${ }^{23}$ (setting $\Delta k=0$, and constant $A_{1,2}(t)$, seed laser phases, and electron-beam energy profile) using the known $R_{56}^{1}$ values and setting $A_{1}=3$ (estimated from measurements of the first stage FERMI emission as a function of dispersive strength ${ }^{16}$ ). It should be emphasized that the spectral properties depend very weakly on the choice of $A_{1}$ when the bunching factor is optimized. For a comparison with the measured FEL spectra, we assumed that the electric field envelope is preserved during amplification in the radiator ${ }^{16}$.

\section{Author contributions}

EA, GDN, DX, LG, and PRR proposed the original idea of an EEHG experiment at FERMI. EA and PRR guided the work and organized the experimental activities. EA, LB, NB, GDN, SDM, BD, WMF, EF, GG, DG, LG, NSM, GMP, $P R R, E R, S S, C S, M T$, and MV conducted the experiment on the accelerator and FEL. EA, WMF, EF, DG, AG, VG, GMP, MP, PRR, and ER analysed the data. EP, EF, EH, GP, PRR, and SR carried out numerical simulations and supported the experiment with theoretical analysis. AA, EA, MB, DC, MCa, PC, IC, AD, BD, MF, GG, FG, LG, SG, $M L, F I, N M, M V, M M, P M, I P N, L P, L R, P R R, R S, C S c, P S, L S, M S, D V, M Z a c, D Z, M Z a n$ contributed to the experimental design and preparation. $P C, A D, G K, P S, I P N$, and $M D$ prepared, operated and optimized the laser systems. FC, MCo, FF, NM, MMal, MMan, PM, LR, and MZan optimized the photon diagnostic used during the experiment. PRR wrote the manuscript draft. All authors participated in the experiment and contributed to improving the final version of the manuscript.

\section{Author affiliations}

\section{Elettra-Sincrotrone Trieste, Area Science Park, 34149 Trieste, Italy}

Primož Rebernik Ribič, Alessandro Abrami, Laura Badano, Maurizio Bossi, Flavio Capotondi, Davide Castronovo, Marco Cautero, Paolo Cinquegrana, Ivan Cudin, Miltcho Boyanov Danailov, Giovanni De Ninno, Alexander Demidovich, Simone Di Mitri, Bruno Diviacco, William M. Fawley, Mario Ferianis, Laura Foglia, Giulio Gaio, Fabio Giacuzzo, Luca Giannessi, Sandi Grulja, Fatma lazzourene, Gabor Kurdi, Marco Lonza, Nicola Mahne, Marco Malvestuto, Michele Manfredda, Claudio Masciovecchio, Najmeh S. Mirian, Ivaylo Petrov Nikolov, 
Giuseppe Maria Penco, Emiliano Principi, Lorenzo Raimondi, Roberto Sauro, Claudio Scafuri, Paolo Sigalotti, Simone Spampinati, Carlo Spezzani, Luca Sturari, Michele Svandrlik, Mauro Trovó, Marco Veronese, Davide Vivoda, Maurizio Zaccaria, Dino Zangrando, Marco Zangrando, and Enrico Massimiliano Allaria

University of Nova Gorica, 5000 Nova Gorica, Slovenia

Primož Rebernik Ribič, Giovanni De Ninno

Paul Scherrer Institut, CH-5232 Villigen PSI, Switzerland

Hans-Heinrich Braun, Eugenio Ferrari, Eduard Prat, Sven Reiche

Department of Engineering and Architecture, Università degli Studi di Trieste, 34127 Trieste, Italy

Niky Bruchon

ISM-CNR, Istituto di Struttura della Materia, LD2 Unit, 34149 Trieste, Italy

Marcello Coreno

Synchrotron SOLEIL, L'Orme des Merisiers, Saint-Aubin, BP 48, 91192 Gif-sur-Yvette, France

Marie Emmanuelle Couprie, Amin Ghaith

SLAC National Accelerator Laboratory, Menlo Park, California 94025, USA

Erik Hemsing

Shanghai Advanced Research Institute, Chinese Academy of Sciences, Shanghai 201210, China

Chao Feng

Institute for Photonics and Nanotechnologies CNR-IFN, Padova, Italy

Fabio Frassetto, Paolo Miotti, Luca Poletto

CEA/DRF/LIDYL, Université Paris-Saclay, Saclay, France

David Garzella

ENEA C.R. Frascati, 00044 Frascati (Roma), Italy

Luca Giannessi

Deutsches Elektronen-Synchrotron DESY, 22607 Hamburg, Germany 
Vanessa Grattoni

Istituto Officina dei Materiali, Consiglio Nazionale delle Ricerche, 34149, Basovizza, TS, Italy

Nicola Mahne, Marco Zangrando

Lawrence Berkeley National Laboratory, Berkeley, CA 94720, USA

Gregory Penn

MAX-IV, Lund University, Lund, Sweden

Mihai Pop

Univ. Lille, CNRS, UMR 8523 - PhLAM - Physique des Lasers Atomes et Molécules, F-59000 Lille, France

Eléonore Roussel

European XFEL, Holzkoppel 4, 22869 Schenefeld, Germany

Takanori Tanikawa

Key Laboratory for Laser Plasmas (Ministry of Education), School of Physics and Astronomy, Shanghai Jiao Tong University, Shanghai 200240, China

Dao Xiang

\section{Corresponding authors}

Primož Rebernik Ribič, Enrico Massimiliano Allaria

\section{Data availability}

The data that support the plots within this paper and other findings of this study are available from the corresponding authors upon reasonable request.

\section{Code availability}

The FEL code Genesis is available at http://genesis.web.psi.ch.

\section{References}

1. McNeil, B. W. J. and Thompson, N. R. X-ray free-electron lasers. Nat. Photon. 4, 814 (2010). 
2. Rebernik Ribic, P. and Margaritondo, G. Status and prospects of $x$-ray free-electron lasers (X-FELs): a simple presentation. J. Phys. Appl. Phys. 45, 213001 (2012).

3. Bonifacio, R., Pellegrini, C. and Narducci, L. M. Collective instabilities and high-gain regime in a free electron laser. Opt. Commun. 50, 373-378 (1984).

4. Ackermann, W. et al. Operation of a free-electron laser from the extreme ultraviolet to the water window. Nat. Photon. 1, 336-342 (2007).

5. Emma, P. et al. First lasing and operation of an angstrom-wavelength free-electron laser. Nat. Photon. 4, 641-647 (2010).

6. Ishikawa, T. et al. A compact X-ray free-electron laser emitting in the sub-angstrom region. Nat Photon 6, 540-544 (2012).

7. Kang, H.-S. et al. Hard X-ray free-electron laser with femtosecond-scale timing jitter. Nat. Photon. 11, 708-713 (2017).

8. Amann, J. et al. Demonstration of self-seeding in a hard-X-ray free-electron laser. Nat. Photon. 6, 693-698 (2012).

9. Ratner, D. et al. Experimental Demonstration of a Soft X-Ray Self-Seeded Free-Electron Laser. Phys. Rev. Lett. 114, 54801 (2015).

10. Lambert, G. et al. Injection of harmonics generated in gas in a free-electron laser providing intense and coherent extreme-ultraviolet light. Nat. Phys. 4, 296-300 (2008).

11. Togashi, T. et al. Extreme ultraviolet free electron laser seeded with high-order harmonic of Ti: Sapphire laser. Opt. Express 19, 317-324 (2011).

12. Ackermann, S. et al. Generation of Coherent 19- and 38-nm Radiation at a Free-Electron Laser Directly Seeded at 38 nm. Phys. Rev. Lett. 111, 114801 (2013).

13. Yu, L. H. Generation of intense uv radiation by subharmonically seeded single-pass freeelectron lasers. Phys. Rev. A 44, 5178-5193 (1991). 
14. Yu, L.-H. et al. High-Gain Harmonic-Generation Free-Electron Laser. Science 289, 932 (2000).

15. Allaria, E. et al. Highly coherent and stable pulses from the FERMI seeded free-electron laser in the extreme ultraviolet. Nat. Photon 6, 699-704 (2012).

16. Gauthier, D. et al. Spectrotemporal Shaping of Seeded Free-Electron Laser Pulses. Phys. Rev. Lett. 115, 114801 (2015).

17. De Ninno, G. et al. Single-shot spectro-temporal characterization of XUV pulses from a seeded free-electron laser. Nat. Commun. 6, 8075 (2015).

18. De Ninno, G., Mahieu, B., Allaria, E., Giannessi, L. and Spampinati, S. Chirped Seeded Free-Electron Lasers: Self-Standing Light Sources for Two-Color Pump-Probe Experiments. Phys. Rev. Lett. 110, 64801 (2013).

19. Ferrari, E. et al. Widely tunable two-colour seeded free-electron laser source for resonant-pump resonant-probe magnetic scattering. Nat. Commun. 7, 10343 (2016).

20. Yu, L.-H. and Ben-Zvi, I. High-gain harmonic generation of soft X-rays with the 'fresh bunch' technique. Free Electron Lasers 1996 Rome Italy Nucl. Instrum. Methods Phys. Res. Sect. Accel. Spectrometers Detect. Assoc. Equip. 393, 96-99 (1997).

21. Allaria, E. et al. Two-stage seeded soft-X-ray free-electron laser. Nat. Photon. 7, 913-918 (2013).

22. Stupakov, G. Using the Beam-Echo Effect for Generation of Short-Wavelength Radiation. Phys. Rev. Lett. 102, 74801 (2009).

23. Xiang, D. and Stupakov, G. Echo-enabled harmonic generation free electron laser. Phys. Rev. Spec. Top. - Accel. Beams 12, 30702 (2009).

24. Penn, G. Stable, coherent free-electron laser pulses using echo-enabled harmonic generation. Phys. Rev. Spec. Top. - Accel. Beams 17, 110707 (2014). 
25. Xiang, D. et al. Demonstration of the Echo-Enabled Harmonic Generation Technique for Short-Wavelength Seeded Free Electron Lasers. Phys. Rev. Lett. 105, 114801 (2010).

26. Zhao, Z. T. et al. First lasing of an echo-enabled harmonic generation free-electron laser. Nat. Photon. 6, 360-363 (2012).

27. Xiang, D. et al. Evidence of High Harmonics from Echo-Enabled Harmonic Generation for Seeding X-Ray Free Electron Lasers. Phys. Rev. Lett. 108, 24802 (2012).

28. Hemsing, E. et al. Highly coherent vacuum ultraviolet radiation at the 15th harmonic with echo-enabled harmonic generation technique. Phys. Rev. Spec. Top. - Accel. Beams 17, 70702 (2014).

29. Hemsing, E. et al. Echo-enabled harmonics up to the 75th order from precisely tailored electron beams. Nat. Photon. 10, 512-515 (2016).

30. Ratner, D., Fry, A., Stupakov, G. and White, W. Laser phase errors in seeded free electron lasers. Phys. Rev. Spec. Top. - Accel. Beams 15, 30702 (2012).

31. Rebernik Ribič, P. et al. Echo-Enabled Harmonic Generation Studies for the FERMI FreeElectron Laser. Photonics 4, (2017).

32. Reiche, S. GENESIS 1.3: a fully 3D time-dependent FEL simulation code. Nucl. Instrum. Methods Phys. Res. Sect. Accel. Spectrometers Detect. Assoc. Equip. 429, 243-248 (1999).

33. Penco, G. et al. Experimental Demonstration of Electron Longitudinal-Phase-Space Linearization by Shaping the Photoinjector Laser Pulse. Phys. Rev. Lett. 112, 44801 (2014).

34. Hemsing, E., Garcia, B., Huang, Z., Raubenheimer, T. and Xiang, D. Sensitivity of echo enabled harmonic generation to sinusoidal electron beam energy structure. Phys. Rev. Accel. Beams 20, 60702 (2017). 
35. Saldin, E. L., Schneidmiller, E. A. and Yurkov, M. V. Klystron instability of a relativistic electron beam in a bunch compressor. Nucl. Instrum. Methods Phys. Res. Sect. Accel. Spectrometers Detect. Assoc. Equip. 490, 1-8 (2002).

36. Borland, M. Modeling of the microbunching instability. Phys. Rev. Spec. Top. - Accel. Beams 11, 30701 (2008).

37. Spampinati, S. et al. Laser heater commissioning at an externally seeded free-electron laser. Phys. Rev. Spec. Top. - Accel. Beams 17, 120705 (2014).

38. Schweigert, I. V. and Mukamel, S. Coherent Ultrafast Core-Hole Correlation Spectroscopy: X-Ray Analogues of Multidimensional NMR. Phys. Rev. Lett. 99, 163001 (2007).

39. Bencivenga, F. et al. Four-wave mixing experiments with extreme ultraviolet transient gratings. Nature 520, 205-208 (2015).

40. Prince, K. C. et al. Coherent control with a short-wavelength free-electron laser. Nat. Photon. 10, 176-179 (2016).

41. Lam, R. K. et al. Soft X-Ray Second Harmonic Generation as an Interfacial Probe. Phys. Rev. Lett. 120, 23901 (2018).

42. Tamasaku, K. et al. Nonlinear Spectroscopy with X-Ray Two-Photon Absorption in Metallic Copper. Phys. Rev. Lett. 121, 83901 (2018).

43. Tanaka, S. and Mukamel, S. Coherent X-Ray Raman Spectroscopy: A Nonlinear Local Probe for Electronic Excitations. Phys. Rev. Lett. 89, 43001 (2002).

44. Penco, G., et al. Optimization of a high brightness photoinjector for a seeded FEL facility. J. Instrum. 8, P05015 (2013).

45. Di Mitri, S. et al. Design and simulation challenges for FERMI@elettra. Nucl. Instrum. Methods Phys. Res. Sect. Accel. Spectrometers Detect. Assoc. Equip. 608, 19-27 (2009). 
46. Penco, G. et al. Time-sliced emittance and energy spread measurements at FERMI@ELETTRA. in Proceedings of FEL2012 (Nara, Japan) WEPD20, 417

47. Craievich, P. et al. Implementation of Radio-Frequency Deflecting Devices for Comprehensive High-Energy Electron Beam Diagnosis. IEEE Trans. Nucl. Sci. 62, 210-220 (2015).

48. Svetina, C. et al. PRESTO, the on-line photon energy spectrometer at FERMI: design, features and commissioning results. J. Synchrotron Radiat. 23, 35-42 (2016).

\section{Acknowledgements}

The authors would like to thank G. Stupakov, S. Bettoni, D. Ratner, G. Marcus, F. Bencivenga, E. Pedersoli, M. Sacchi, C. Callegari, Z. Huang, T. Raubenheimer, and A. Zholents for useful discussions. The authors also acknowledge the continuous support of R. Godnig, R. Bracco, R. Visintini, and the FERMI - Elettra operator group during the experiment. This work was supported in part by the Director, Office of Science, Office of Basic Energy Sciences, of the U.S. Department of Energy under Contract Nos. DE-AC02-76SF00515 and DE-AC0205CH11231 and award No. 2017-SLAC-100382. DG was supported by an Outgoing CEA fellowship from the CEA-Enhanced Eurotalents program, co-funded by FP7 Marie-Skłodowska-Curie COFUND program (Grant Agreement 600382). 